\title{
Connectivity of the frontal and anterior insular network: a cortico-cortical evoked potential study
}

\author{
Rei Enatsu, MD, PhD, 1,2 Jorge Gonzalez-Martinez, MD, PhD, ${ }^{1}$ Juan Bulacio, MD,1 \\ John C. Mosher, PhD, ${ }^{1}$ Richard C. Burgess, MD, PhD, ${ }^{1}$ Imad Najm, MD, ${ }^{1}$ and Dileep R. Nair, MD ${ }^{1}$ \\ ${ }^{1}$ Epilepsy Center, Neurological Institute, Cleveland Clinic, Cleveland, Ohio; and ${ }^{2 D e p a r t m e n t ~ o f ~ N e u r o s u r g e r y, ~ S a p p o r o ~ M e d i c a l ~}$ \\ University, Sapporo, Japan
}

\begin{abstract}
OBJECTIVE The frontal and insular fiber network in humans remains largely unknown. This study investigated the connectivity of the frontal and anterior insular network in humans using cortico-cortical evoked potential (CCEP).

METHODS This retrospective analysis included 18 patients with medically intractable focal epilepsy who underwent stereoelectroencephalography and CCEP. Alternating $1-\mathrm{Hz}$ electrical stimuli were delivered to parts of the frontal lobe and anterior insula (prefrontal cortex [PFC], ventrolateral and dorsolateral premotor area [VPM and dPM, respectively], presupplementary motor area [pre-SMA], SMA, frontal operculum, and anterior insula). A total of 40-60 stimuli were averaged in each trial to obtain CCEP responses. The distribution of CCEP was evaluated by calculating the root mean square of CCEP responses.

RESULTS Stimulation of the PFC elicited prominent CCEP responses in the medial PFC and PMs over the ipsilateral hemisphere. Stimulation of the vPM and dPM induced CCEP responses in the ipsilateral frontoparietal areas. Stimulation of the pre-SMA induced CCEP responses in the ipsilateral medial and lateral frontal areas and contralateral pre-SMA, whereas stimulation of the SMA induced CCEP responses in the bilateral frontoparietal areas. Stimulation of the frontal operculum induced CCEP responses in the ipsilateral insula and temporal operculum. CCEPs were observed in the ipsilateral medial, lateral frontal, and frontotemporal operculum in the anterior insular stimulation. Stimulation of the VPM and SMA led to the network in the dominant hemisphere being more developed.
\end{abstract}

CONCLUSIONS Various regions within the frontal lobe and anterior insula were linked to specific ipsilateral and contralateral regions, which may reflect distinct functional roles.

http://thejns.org/doi/abs/10.3171/2015.6.JNS15622

KEY WORDS brain network; frontal lobe; insula; stereoelectroencephalography; evoked potential; epilepsy; functional neurosurgery

$\mathrm{T}$ $\checkmark \mathrm{HE}$ functional roles of the frontal lobe, including motor execution, attention and memory, executive cognition, social behavior, and consciousness, have already been elucidated. ${ }^{7}$ The insula is also functionally involved in cardiac rhythm and arterial blood pressure control, as well as in visceromotor control, viscerosensitive functions, and the processing of nociceptive input. ${ }^{6,26}$ This wide range of abilities relies on multiple fiber networks. Anatomical and functional relationships in the frontal and insular fiber network remain largely unknown in humans. Therefore, a clearer understanding of these relationships will be useful not only for providing an insight into human brain functions, but also for evaluating focal epilepsy in- volving these areas. Difficulties have been associated with examining frontal and insular epilepsy due to complicated connectivity patterns, the deep anatomical location of seizure onset, and rapid seizure spread. ${ }^{1,3,4,16}$ Therefore, expanding our knowledge on the frontal and insular network is important for presurgical evaluations of patients with pharmacoresistant epilepsy arising within these areas.

Electrical stimulation was recently introduced as an in vivo test to track the various human brain networks. ${ }^{2,21,22,28}$ We previously developed a technique termed cortico-cortical evoked potential (CCEP) to examine human brain networks in vivo. ${ }^{22}$ In the present study, we attempted to identify functional connections in the frontal and anterior

ABBREVIATIONS CCEP = cortico-cortical evoked potential; $\mathrm{dPM}=$ dorsolateral premotor area; $\mathrm{PCL}=$ paracentral lobule; $\mathrm{PFC}=$ prefrontal cortex; $\mathrm{RMS}=$ root mean square; SEEG = stereoelectroencephalography; SLF = superior longitudinal fasciculus; SMA = supplementary motor area; VPC = vertical posterior-commissural; vPM = ventrolateral premotor area.

SUBMITTED March 18, 2015. ACCEPTED June 18, 2015.

INCLUDE WHEN CITING Published online December 18, 2015; DOI: 10.3171/2015.6.JNS15622. 
insular network using CCEP in patients implanted with stereoelectroencephalography (SEEG) electrodes.

\section{Methods \\ Patient Population}

This retrospective analysis included 18 patients $(6$ females) with medically intractable focal epilepsy who underwent SEEG evaluations at the Cleveland Clinic Epilepsy Center beginning in 2009. Seventeen patients were right-handed and 1 was left-handed. Their ages ranged from 15 to 58 years (median 26 years) and age at seizure onset ranged from 6 months to 37 years (median 11 years). All patients underwent CCEP with stimulation of parts of the frontal or insular lobe (the prefrontal cortex [PFC], ventrolateral and dorsolateral premotor area [vPM and dPM, respectively], presupplementary motor area [pre-SMA], SMA, frontal operculum, and anterior insula) as part of their routine invasive neurophysiological analysis for clinical purposes, such as understanding ictal propagation.

The following regions were stimulated for the CCEP analysis. The right PFC was stimulated in 2 patients and the left PFC in 3 patients. The right $\mathrm{VPM}$ was stimulated at 2 locations in 2 patients and the left vPM was stimulated at 3 locations in 2 patients. The right dPM was stimulated at 5 sites in 4 patients and the left dPM at 4 sites in 3 patients. The pre-SMA was stimulated at 6 locations in 4 patients on the right side and at 6 locations in 4 patients on the left side. There were 5 stimulation sites in the right SMA in 4 patients, 5 stimulation sites in the left SMA in 4 patients, 6 stimulation sites in the right frontal operculum in 3 patients, and 6 stimulation sites in the left operculum in 3 patients. The anterior insula was stimulated at 5 locations in 3 patients on the right and at 4 locations in 2 patients on the left. The number of recording electrodes in each area is shown in Tables 1-4. This study was approved by the institutional review board committee of Cleveland Clinic.

\section{Implantation of SEEG Electrodes}

Implantation targets were determined based on a clinically generated preimplantation hypothesis for localization of the epileptogenic zone. Depth electrode targeting and trajectory were determined using standard stereotactic software (iPlan; Brainlab) or a robotic system (ROSA; Medtech). The planned trajectory was reviewed to verify that no vessels or other important structures were at risk for injury, and was modified if necessary. Under general anesthesia, the electrodes were inserted consecutively in an orthogonal or oblique manner in relation to the midline vertical plane. The number of implanted SEEG electrodes ranged from 12 to 17 (median 14) per patient. The electrodes consisted of 10-12 cylindrical 2.5-mm-long platinum contacts with a diameter of $1.1 \mathrm{~mm}$ (Integra Epilepsy; Integra LifeSciences Corp.) or 10 cylindrical 2.3-mm-long platinum contacts with a diameter of 0.89 $\mathrm{mm}$ (Ad-Tech). The patients underwent a postoperative high-resolution $\mathrm{CT}$ scan after implantation to verify the exact location of each contact and to detect any postoperative complications.

The PM, SMA, and pre-SMA were defined similar to previous descriptions.11,14,23 The rostral border of the PM was defined as 30-35, 15-30, and $15-20 \mathrm{~mm}$ rostral to the precentral sulcus in the superior, middle, and inferior frontal gyri, respectively. ${ }^{11,23}$ The medial PM, which is above the cingulate sulcus, was subdivided into rostral pre-SMA and caudal SMA parts. The vertical anterior-commissural line was used as a landmark to differentiate between preSMA and SMA. ${ }^{10,23}$ The vertical posterior-commissural (VPC) line was also used as an anatomical landmark to differentiate between the SMA and paracentral lobule (PCL).$^{10}$ The remaining area of the frontal lobe anterior to the PM was defined as the PFC., ${ }^{9,18}$ The inferior choroidal point was used as a landmark to separate the anterior and posterior hippocampus. ${ }^{20}$ The cingulate gyrus was divided into anterior and posterior areas. The anterior cingulate gyrus was located rostral to the VPC line and the posterior cingulate gyrus was located caudal to the VPC line..$^{15}$ The central sulcus of the insula was used to divide the insula into anterior and posterior parts. ${ }^{26}$

\section{CCEP Recording and Acquisition of CCEP Waveforms}

The present study was performed extraoperatively with the patient in a resting state without any special tasks after the standard presurgical evaluation and restart of antiepileptic medications. Parts of the frontal and insular network were stimulated through 2 adjacent contacts in a bipolar manner after their positions were confirmed on postoperatively reconstructed MR images. The cortical contacts were chosen for both stimulation and recording. The electrical stimulus consisted of a constant current, square-wave pulse with a $0.3-\mathrm{msec}$ duration and pulse frequency of $1 \mathrm{~Hz}$ with alternating polarity. The current intensity started at $2 \mathrm{~mA}$ and was increased by $2 \mathrm{~mA}$ in stepwise increments to $8 \mathrm{~mA}$. Taking into account the surface area of the SEEG electrode, the maximum intensity was set to $8 \mathrm{~mA}$ to adjust the charge density to our protocol of cortical stimulation. ${ }^{22,24}$

Forty to 60 stimuli were delivered in each session. To confirm its reproducibility, 8-mA sessions were performed twice. Electrical pulses were generated with Grass S88 (SUI-7; Astro-Med, Inc.) and raw data were recorded from SEEG electrodes on a digital EEG machine (NeuroWorkbench V03-35; Nihon Kohden America, Inc.). The sampling rate was set at $1 \mathrm{kHz}$. CCEPs were obtained using an offline averaging time locked to the stimulus onset with a $1-\mathrm{Hz}$ low-cut filter and a $300-\mathrm{Hz}$ high-cut filter. The average time window was $400 \mathrm{msec}$ with a 100 -msec prestimulus period. After averaging, the epoch distorted by the definite artifact was discarded from the analysis. In each session, 40-60 responses were averaged. These averaged CCEP data were reviewed using MATLAB R2006b version 7.3.0.267 (MathWorks, Inc.).

\section{Quantitative Analysis of CCEP Responses}

We previously reported that the CCEP response consisted of early (N1) and late (N2) negative deflections. ${ }^{22}$ However, CCEP responses may also have various morphologies with wide-range peak latencies. Variations in the absolute amplitudes and latencies of the recorded peaks led to difficulties in quantifying CCEP responses. Therefore, to quantify the strength of responses, we calculated the root mean square (RMS) for each response, which is defined 


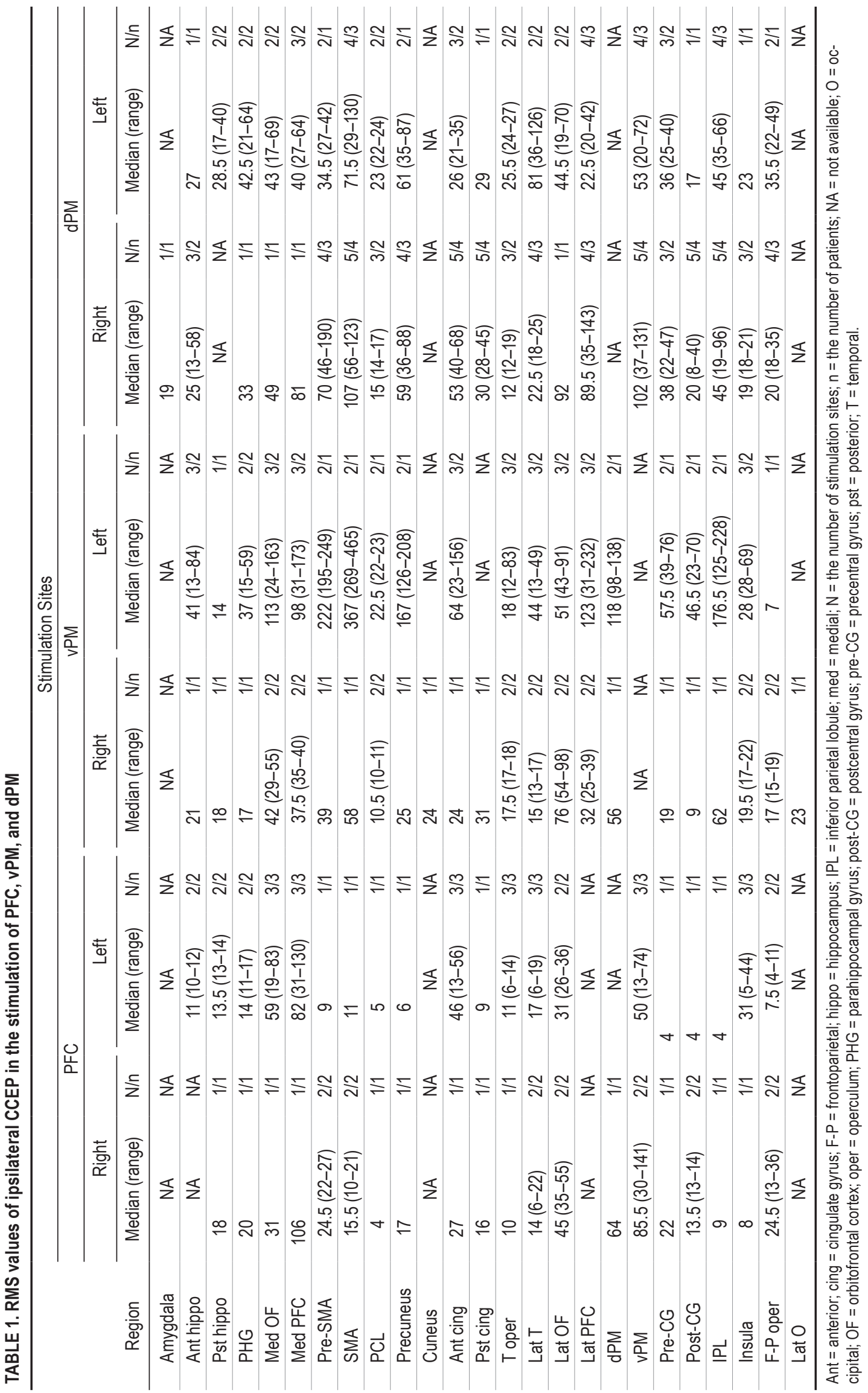




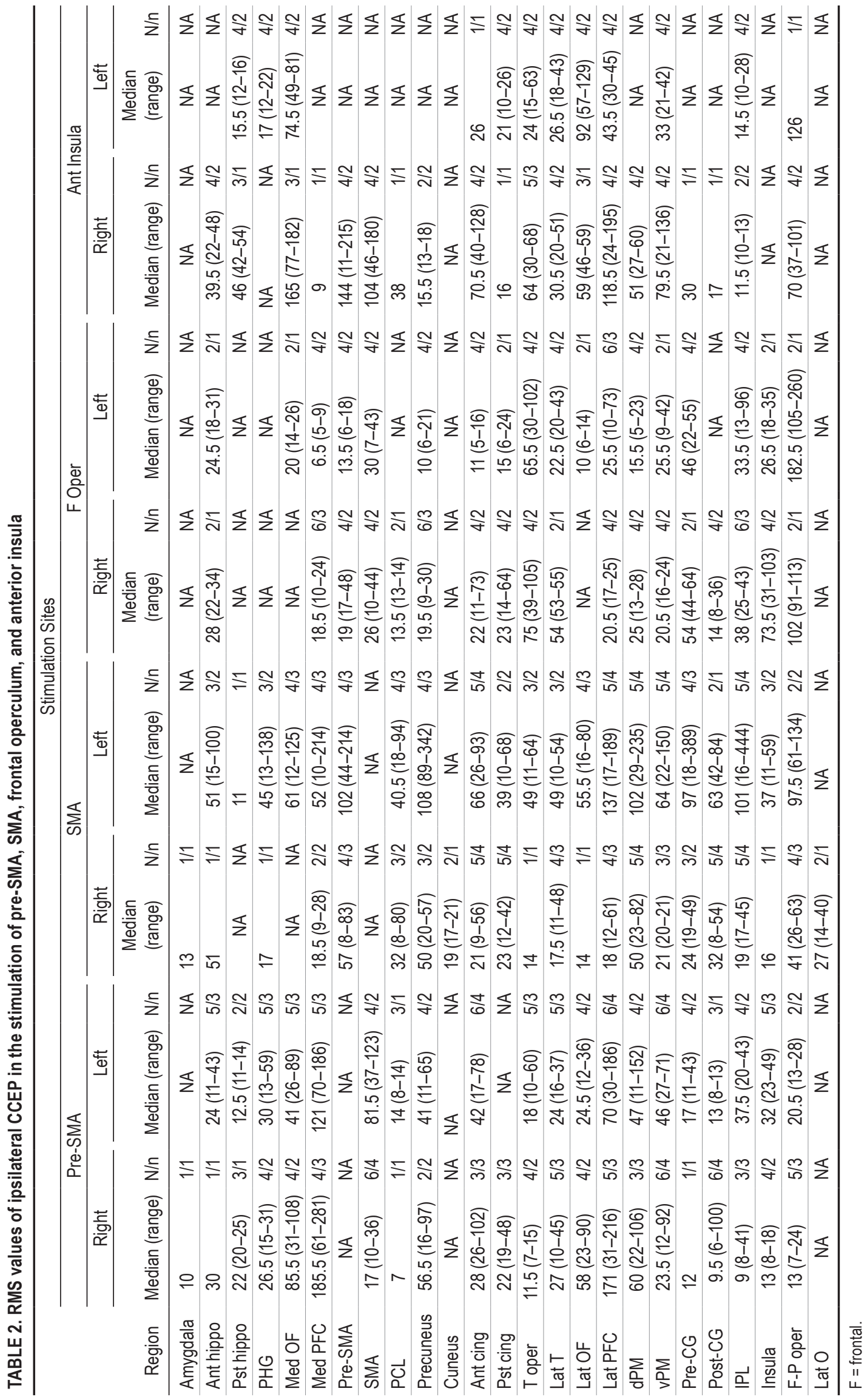


TABLE 3. RMS values of contralateral CCEP in the stimulation of PFC, vPM, and dPM

\begin{tabular}{|c|c|c|c|c|c|c|c|c|c|c|c|c|}
\hline \multirow[b]{4}{*}{ Region } & \multicolumn{12}{|c|}{ Stimulation Sites } \\
\hline & \multicolumn{4}{|c|}{ PFC } & \multicolumn{4}{|c|}{ vPM } & \multicolumn{4}{|c|}{$\mathrm{dPM}$} \\
\hline & \multicolumn{2}{|c|}{ Right } & \multicolumn{2}{|c|}{ Left } & \multicolumn{2}{|c|}{ Right } & \multicolumn{2}{|c|}{ Left } & \multicolumn{2}{|c|}{ Right } & \multicolumn{2}{|l|}{ Left } \\
\hline & $\begin{array}{l}\text { Median } \\
\text { (range) }\end{array}$ & $N / n$ & $\begin{array}{l}\text { Median } \\
\text { (range) }\end{array}$ & $\mathrm{N} / \mathrm{n}$ & $\begin{array}{l}\text { Median } \\
\text { (range) }\end{array}$ & $\mathrm{N} / \mathrm{n}$ & $\begin{array}{l}\text { Median } \\
\text { (range) }\end{array}$ & $\mathrm{N} / \mathrm{n}$ & $\begin{array}{l}\text { Median } \\
\text { (range) }\end{array}$ & $N / n$ & Median (range) & $\mathrm{N} / \mathrm{n}$ \\
\hline Amygdala & NA & NA & NA & NA & NA & NA & NA & NA & NA & NA & NA & NA \\
\hline Ant hippo & NA & NA & NA & NA & NA & NA & NA & NA & NA & NA & 13 & $1 / 1$ \\
\hline Pst hippo & NA & NA & NA & NA & NA & NA & NA & NA & NA & NA & NA & NA \\
\hline PHG & NA & NA & NA & NA & NA & NA & NA & NA & NA & NA & NA & NA \\
\hline Med OF & NA & NA & NA & NA & NA & NA & NA & NA & NA & NA & NA & NA \\
\hline Med PFC & NA & NA & NA & NA & NA & NA & NA & NA & 13 & $1 / 1$ & $18(9-27)$ & $2 / 1$ \\
\hline Pre-SMA & NA & NA & NA & NA & NA & NA & NA & NA & 22 & $1 / 1$ & $32(17-47)$ & $2 / 1$ \\
\hline SMA & NA & NA & NA & NA & NA & NA & NA & NA & 19 & $1 / 1$ & $33(28-38)$ & $2 / 1$ \\
\hline PCL & NA & NA & NA & NA & NA & NA & NA & NA & NA & NA & NA & NA \\
\hline Precuneus & NA & NA & NA & NA & NA & NA & NA & NA & 23 & $1 / 1$ & $50.5(31-70)$ & $2 / 1$ \\
\hline Cuneus & NA & NA & NA & NA & NA & NA & NA & NA & NA & NA & NA & NA \\
\hline Ant cing & NA & NA & NA & NA & NA & NA & NA & NA & 22 & $1 / 1$ & $28(12-29)$ & $3 / 2$ \\
\hline Pst cing & NA & NA & NA & NA & NA & NA & NA & NA & NA & NA & $25(11-39)$ & $2 / 1$ \\
\hline T oper & NA & NA & NA & NA & NA & NA & NA & NA & NA & NA & NA & NA \\
\hline Lat T & NA & NA & NA & NA & NA & NA & NA & NA & NA & NA & NA & NA \\
\hline Lat OF & NA & NA & NA & NA & NA & NA & NA & NA & NA & NA & NA & NA \\
\hline Lat PFC & NA & NA & NA & NA & NA & NA & NA & NA & 8 & $1 / 1$ & 25 (14-39) & $3 / 2$ \\
\hline dPM & NA & NA & NA & NA & NA & NA & NA & NA & 9 & $1 / 1$ & $30.5(21-40)$ & $2 / 1$ \\
\hline vPM & NA & NA & NA & NA & NA & NA & NA & NA & 18 & $1 / 1$ & $61(37-85)$ & $2 / 1$ \\
\hline Pre-CG & NA & NA & NA & NA & NA & NA & NA & NA & 14 & $1 / 1$ & NA & NA \\
\hline Post-CG & NA & NA & NA & NA & NA & NA & NA & NA & NA & NA & $28.5(17-40)$ & $2 / 1$ \\
\hline IPL & NA & NA & NA & NA & NA & NA & NA & NA & 20 & $1 / 1$ & $48.5(36-61)$ & $2 / 1$ \\
\hline Insula & NA & NA & NA & NA & NA & NA & NA & NA & NA & NA & NA & NA \\
\hline F-P oper & NA & NA & NA & NA & NA & NA & NA & NA & 18 & $1 / 1$ & NA & NA \\
\hline Lat O & NA & NA & NA & NA & NA & NA & NA & NA & NA & NA & NA & NA \\
\hline
\end{tabular}

as the square root of the mean of the squares of amplitude value ( $\mu \mathrm{A})$ (1-msec slide) from $15 \mathrm{msec}$ to $300 \mathrm{msec}$. This measurement was adapted to the limbic network in our previous studies. ${ }^{13,19}$ The initial $15-\mathrm{msec}$ period from the stimulus was obscured by stimulus artifacts; therefore, we excluded the initial $15 \mathrm{msec}$ from the stimulus onset. We analyzed CCEP data stimulated at $8 \mathrm{~mA}$ in all patients. To illustrate the distribution of activity over the cortex, a circle map was used based on the median RMS value in all patients, in which the diameter of the circle at each electrode represented the RMS value of CCEP activities.

\section{Results}

Sample waveforms of CCEPs are shown in Fig. 1 and the distribution of CCEPs is shown in Figs. 2-4. The RMS values of CCEP responses are shown in Tables 1-4. Tables 1 and 2 show the hemispheric responses ipsilateral to the electrical stimulation and Tables 3 and 4 represent the contralateral responses.

Figure 1 shows sample waveforms of CCEPs in the vPM stimulation and pre-SMA stimulation. As shown in the example of CCEP data taken from the left vPM stimulation in 1 subject, CCEP responses were observed in the ipsilateral medial and lateral PFC, pre-SMA, orbitofrontal cortex, precentral gyrus, and inferior parietal lobule (Fig. 1A). In the left pre-SMA stimulation, CCEP responses were obtained in the ipsilateral medial and lateral PFC, vPM, hippocampus, precuneus, and inferior parietal lobule (Fig. 1B).

\section{PFC and PM Stimulation}

As a group, stimulation of the right PFC $(\mathrm{N}=2)$ elicited prominent CCEP responses with an RMS value higher than 50 in the medial PFC and PMs over the ipsilateral hemisphere (Fig. 2A). The left PFC stimulation $(\mathrm{N}=3)$ induced prominent CCEP responses in the medial PFC, medial orbitofrontal cortex, and PMs over the ipsilateral hemisphere (Fig. 2B).

The right $v P M$ stimulation $(\mathrm{N}=2)$ induced CCEP responses in the ipsilateral lateral orbitofrontal cortex, dPM, inferior parietal lobule, and SMA (Fig. 2C), whereas the left vPM stimulation $(\mathrm{N}=3)$ induced CCEP responses in the broader areas including the ipsilateral lateral and medial orbitofrontal cortex, lateral and medial PFC, preSMA, SMA, anterior cingulate gyrus, dPM, precentral gyrus, precuneus, and inferior parietal lobule (Fig. 2D). 


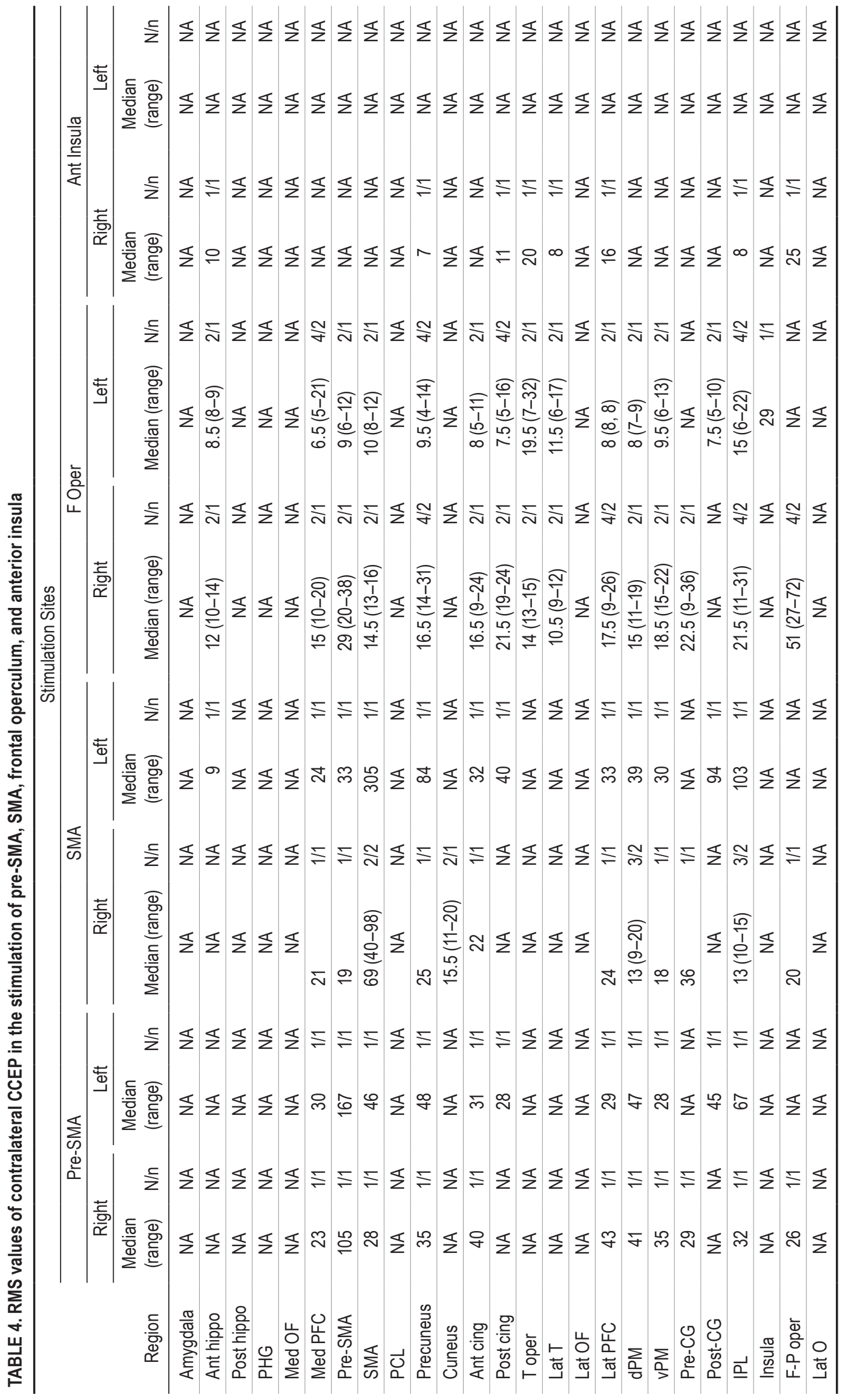



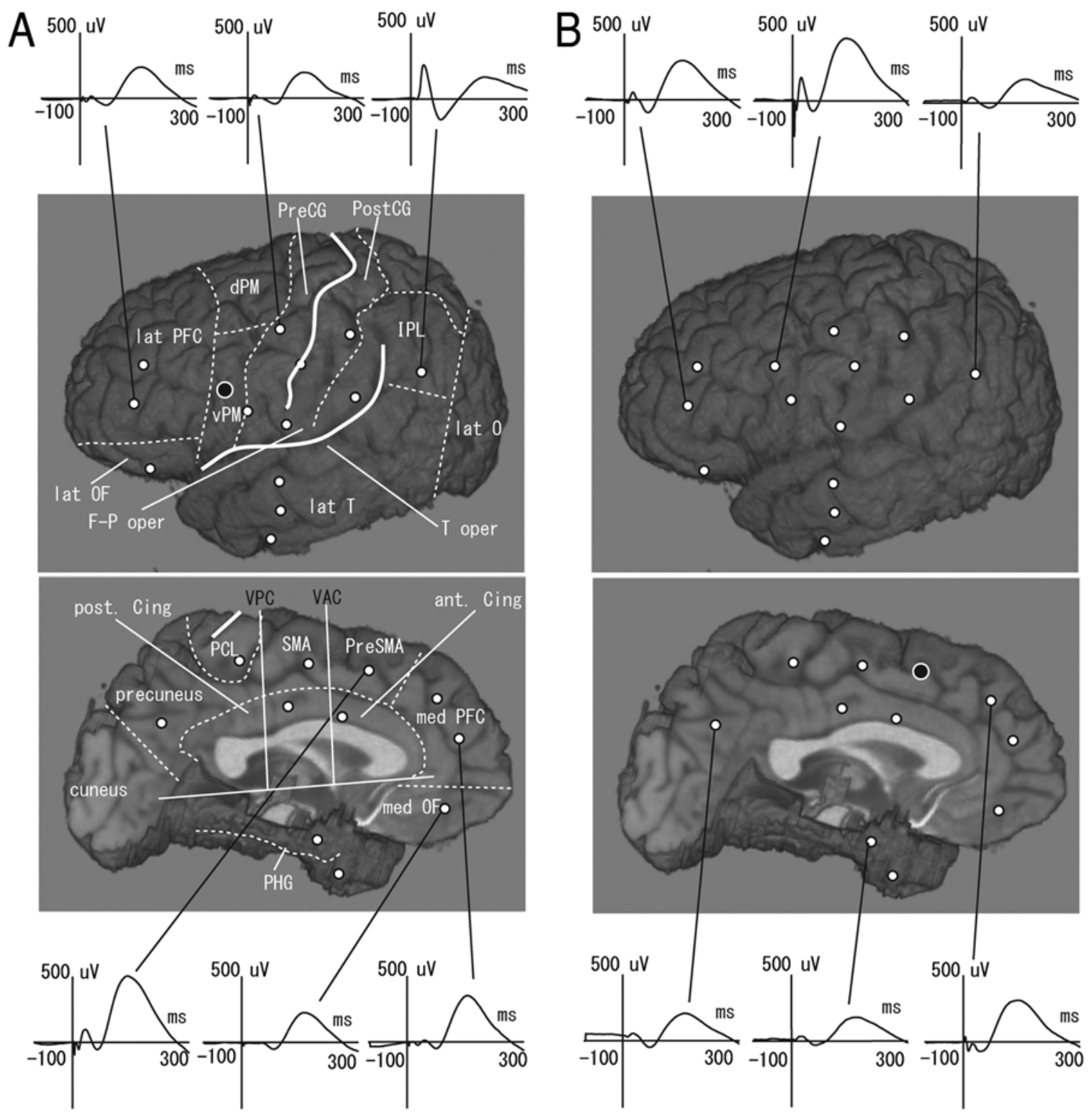

FIG. 1. Borders of each area and examples of CCEP waveforms in the left vPM stimulation (A) and left pre-SMA stimulation (B). The black dot indicates the stimulation site and the white dots the recording sites. ant = anterior; cing = cingulate gyrus; F-P $=$ frontoparietal; $I P L=$ inferior parietal lobule; med = medial; $\mathrm{O}=$ occipital; $\mathrm{OF}=$ orbitofrontal cortex; oper = operculum; $\mathrm{PHG}=$ parahippocampal gyrus; post = posterior; post-CG = postcentral gyrus; pre-CG = precentral gyrus, $\mathrm{T}=$ temporal; $\mathrm{VAC}=$ vertical anterior-commissural line.

The right dPM stimulation $(\mathrm{N}=5)$ induced CCEP responses in the ipsilateral lateral orbitofrontal cortex, lateral and medial PFC, vPM, pre-SMA, SMA, anterior cingulate gyrus, and precuneus (Fig. 2E). The left dPM stimulation $(\mathrm{N}=4)$ induced CCEP responses in the lateral temporal cortex, vPM, SMA, precuneus, and contralateral vPM and precuneus (Fig. 2F).

\section{Pre-SMA and SMA Stimulation}

When the right pre-SMA was stimulated $(\mathrm{N}=6)$, CCEP responses were observed in the ipsilateral lateral and medial orbitofrontal cortex, lateral and medial PFC, dPM, precuneus, and contralateral pre-SMA (Fig. 3A). In the left pre-SMA stimulation $(\mathrm{N}=6)$, CCEP responses were obtained in the ipsilateral lateral and medial PFC, SMA, contralateral pre-SMA, and inferior parietal lobule (Fig. 3B).

The right SMA stimulation $(\mathrm{N}=5)$ induced CCEP responses in the ipsilateral dPM, pre-SMA, precuneus, anterior hippocampus, and contralateral SMA (Fig. 3C). The left SMA stimulation $(\mathrm{N}=5)$ showed broader CCEP responses in the lateral and medial orbitofrontal cortex, lateral and medial PFC, pre-SMA, anterior cingulate gyrus, dPM, vPM, precentral gyrus, postcentral gyrus, frontoparietal operculum, inferior parietal lobule, anterior hippocampus, and precuneus over the ipsilateral hemisphere and in the SMA, precuneus, postcentral gyrus, and inferior parietal lobule over the contralateral hemisphere (Fig. 3D). 
RMS 200

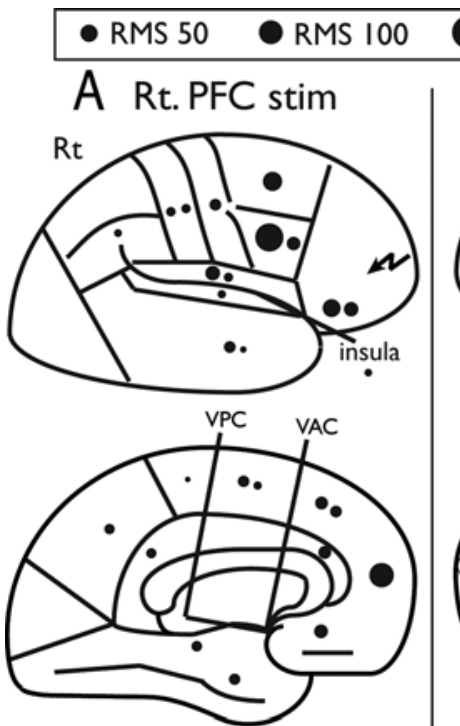

A Rt. PFC stim

E Rt. dPM stim
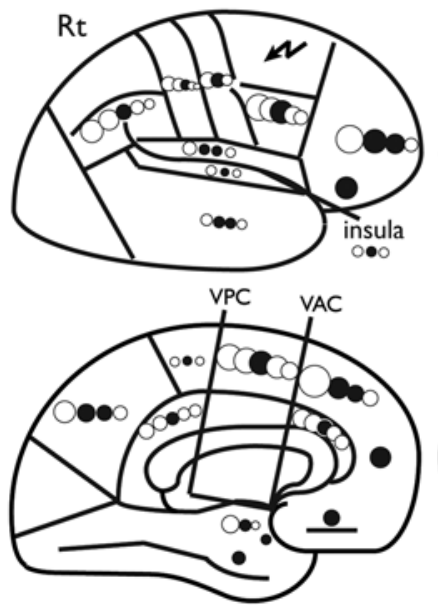

B Lt. PFC stim
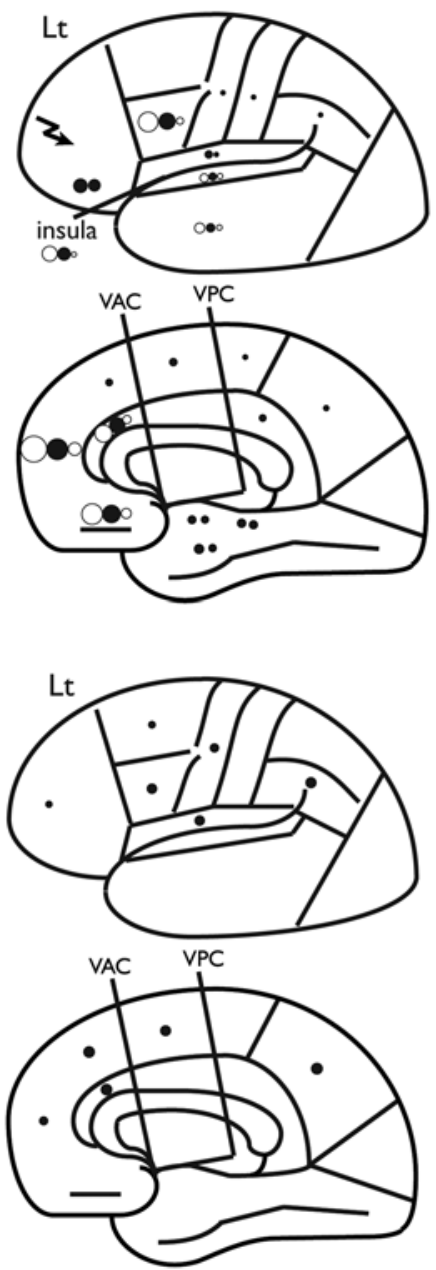

C Rt.vPM stim
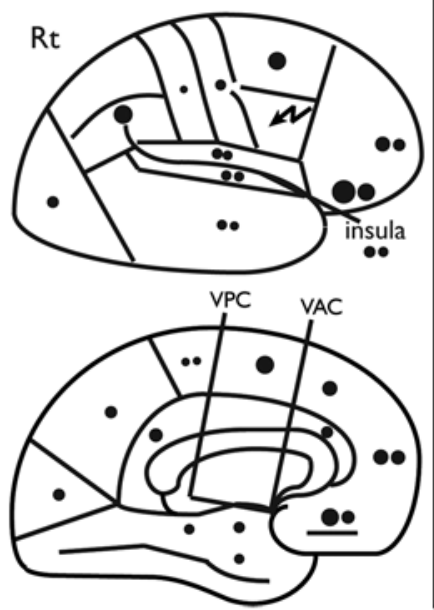

F Lt. dPM stim
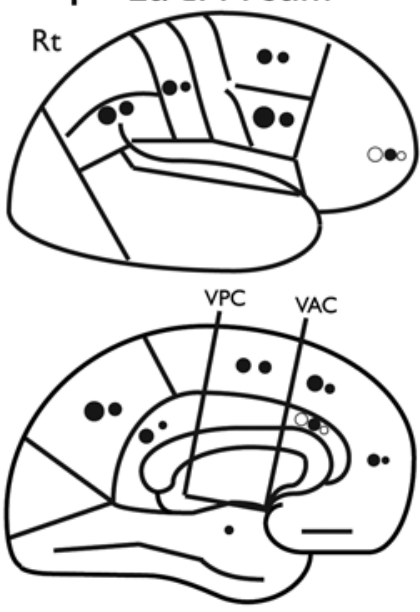

D Lt.vPM stim
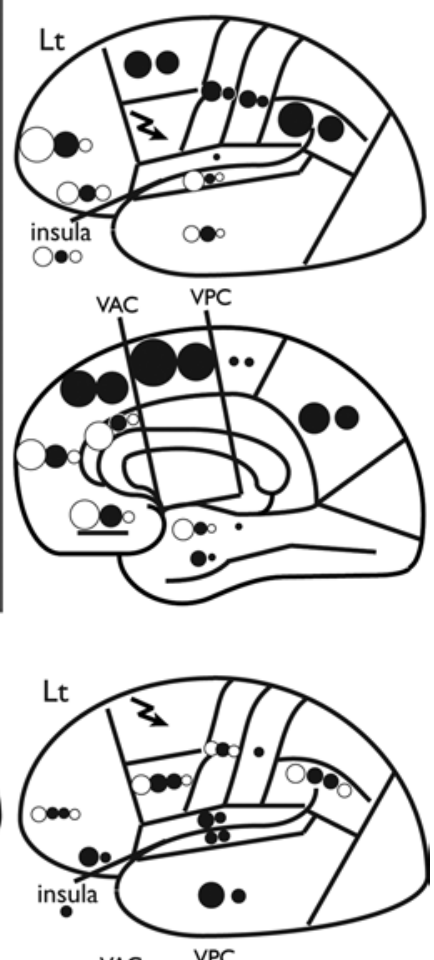

FIG. 2. Circle maps of RMS values of CCEPs in the PFC stimulation (A) right $(N=2)$ and $(B)$ left $(N=3)$, vPM stimulation (C) right $(N=2)$ and $(D)$ left $(N=3)$, and dPM stimulation $(E)$ right $(N=5)$ and $(F)$ left $(N=4)$. The diameter of the circle represents the RMS value of CCEP activities. Blank parcellation (empty circles) indicate "not available" and black circles indicate the median value of RMS in each parcellation. stim = stimulation.

\section{Frontal Operculum and Insula Stimulation}

The CCEP responses were recorded in all patients who underwent the right frontal operculum stimulation $(\mathrm{N}=$ 6 ), induced from the parietal operculum, temporal operculum, insula, lateral temporal cortex, and precentral gyrus on the ipsilateral side and contralateral frontal operculum (Fig. 4A). The left frontal operculum stimulation $(\mathrm{N}=6)$ elicited CCEP responses in the ipsilateral parietal and temporal operculum (Fig. 4B).

In the right insular stimulation $(\mathrm{N}=5)$, CCEPs were observed in the ipsilateral lateral and medial orbitofrontal cortex, lateral PFC, anterior cingulate gyrus, pre-SMA, SMA, dPM, vPM, frontal operculum, and temporal operculum (Fig. 4C). The left insular stimulation $(\mathrm{N}=4)$ elicited CCEP responses in the ipsilateral lateral and medial orbitofrontal cortex and in the frontal operculum (Fig. 4D).

\section{Discussion}

The results of the present study demonstrated the func- tional (electrophysiological) connectivity between various components of the human frontal and insular network, and provided an anatomical framework for local connectivity in the frontal lobes and insula that may be used in future clinical research.

Our stimulation induced evoked potentials, which revealed electrophysiological connections from the PFC to the medial PFC and PM over the ipsilateral hemisphere. The PFC is involved in higher functions in humans such as working memory, episodic memory retrieval, mentalizing, monitoring self-generated choices, allocating attention between simultaneous tasks, and prospectively coding and deferring goals in multitasking.? A previous diffusion tractography and postmortem dissection study suggested that the frontal longitudinal system connected the dorsolateral cortex of the PM and PFC. ${ }^{7}$ Furthermore, the frontomarginal tract runs beneath the frontomarginal sulcus and connects medial and lateral regions of the frontopolar cortex. These anatomical structures may explain the connectivity patterns from the PFC observed in our study. 

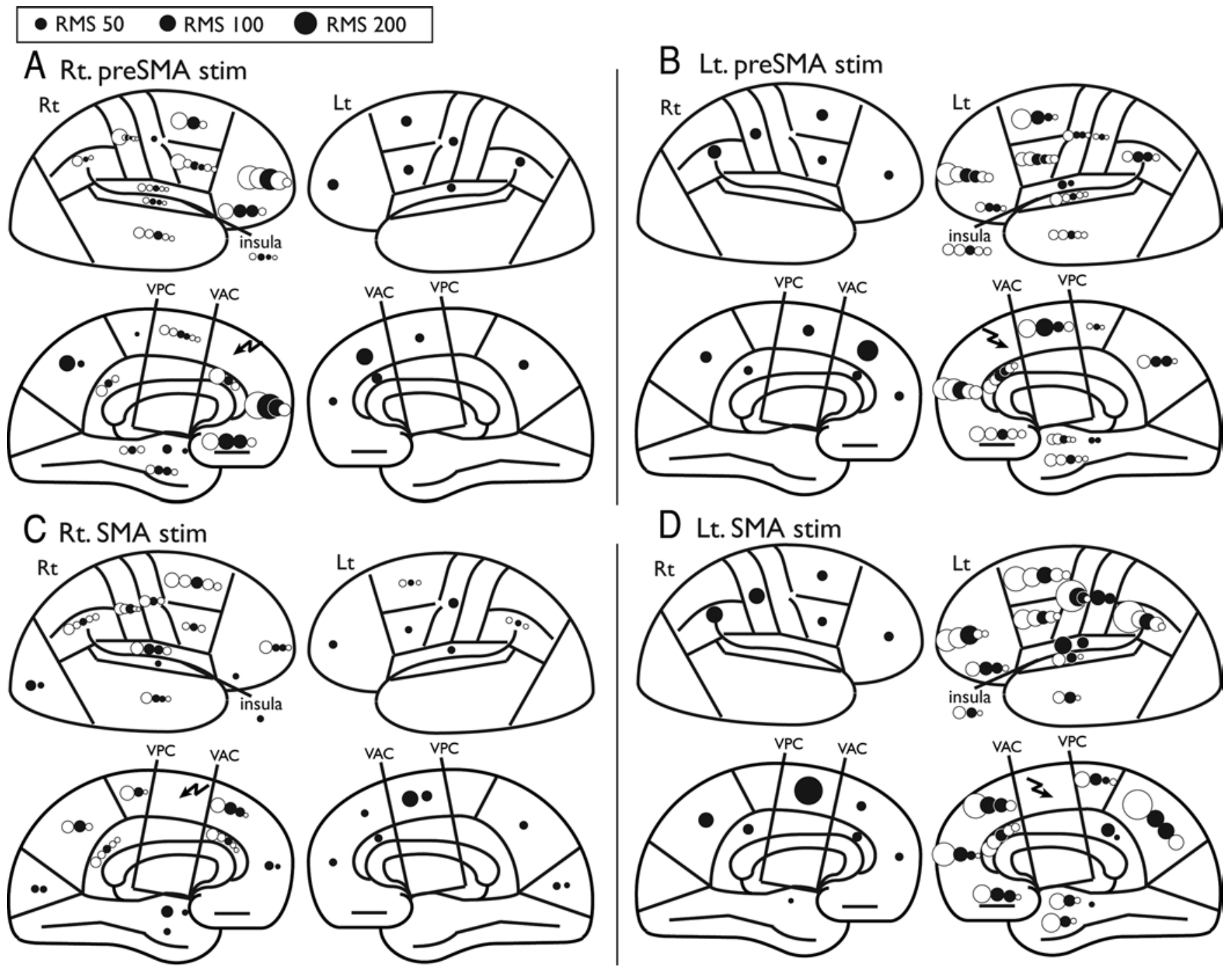

FIG. 3. Circle maps of RMS values of CCEPs in the pre-SMA stimulation (A) right $(N=6)$ and $(B)$ left $(N=6)$, and SMA stimulation (C) right $(\mathrm{N}=5)$ and (D) left $(\mathrm{N}=5)$. The diameter of the circle represents the RMS value of CCEP activities. Blank parcellation (empty circles) indicate "not available" and black circles indicate the median value of RMS in each parcellation.

The vPM stimulation induced CCEP responses in the ipsilateral PFC, orbitofrontal cortex, dPM, inferior parietal lobule, and SMA, whereas the APM stimulation induced CCEP responses in the ipsilateral vPM, SMA, and precuneus. The frontal aslant tract connects the SMA and pre-SMA with the inferior frontal gyrus. The frontal longitudinal system connects the dorsolateral cortex of the PM and PFC. ${ }^{7}$ The superior longitudinal fasciculus (SLF) consists of 3 components, connecting specific frontal areas with the posterior parietal cortex. SLF I links the superior parietal lobule with the PMs and SMA; SLF II courses between the caudal part of the inferior parietal lobule and the intraparietal sulcus and the posterior prefrontal cortices; and SLF III extends from the rostral inferior parietal lobule to the ventral part of the PM and PFC..$^{25}$ These subcomponents are relevant to the higher-order control of body-centered actions, spatial attention, and action imitation, respectively. These anatomical structures also correlate with our results on connectivity from the PM to frontoparietal area.
The present results revealed connections from the preSMA to the ipsilateral medial and lateral frontal areas and contralateral pre-SMA, whereas the SMA had connections to the bilateral frontoparietal areas. The frontal aslant tract connected the SMA and pre-SMA with the lateral PM areas. The SMA was previously shown to connect with the superior parietal lobule through SLF I, as discussed above. ${ }^{25}$ Projections from the pre-SMA and SMA to the ipsilateral frontoparietal areas may reflect these anatomical bundles. Furthermore, these medial frontal areas have been shown to possess interhemispheric connections, as described previously. ${ }^{8}$ Our results revealed new connections from the SMA to the anterior hippocampus, which have not yet been described and are of interest with respect to the functional relationship between behavior and memory. Further cases are needed to confirm these novel connections.

The frontal operculum had connections to the ipsilateral insula and temporal operculum, whereas the insula had connections to the ipsilateral medial, lateral frontal, and frontotemporal operculum. Previous studies reported the 


\section{$\bullet$ RMS $50 \bigcirc$ RMS $100 \bigcirc$ RMS 200}

A Rt. frontal operculum stim
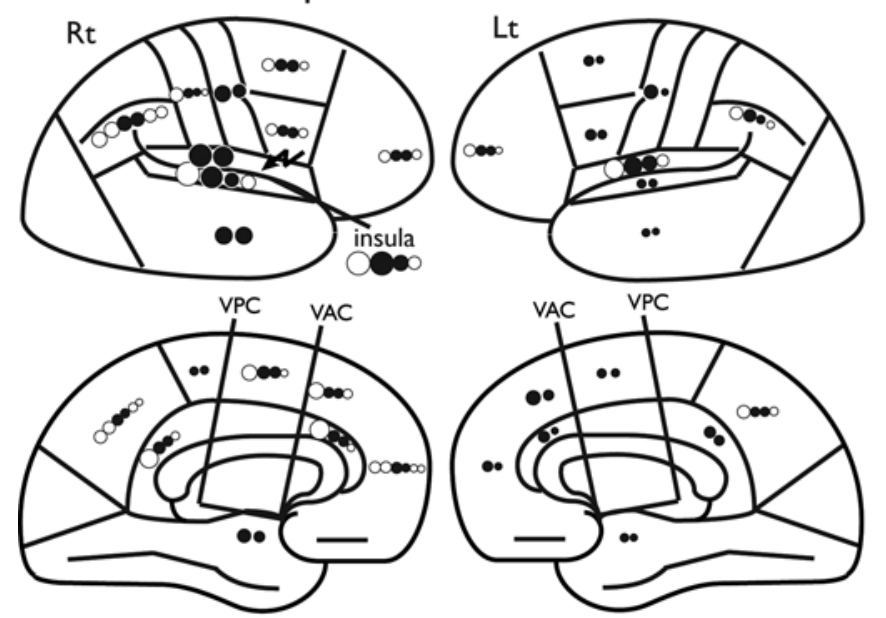

C Rt. insula stim
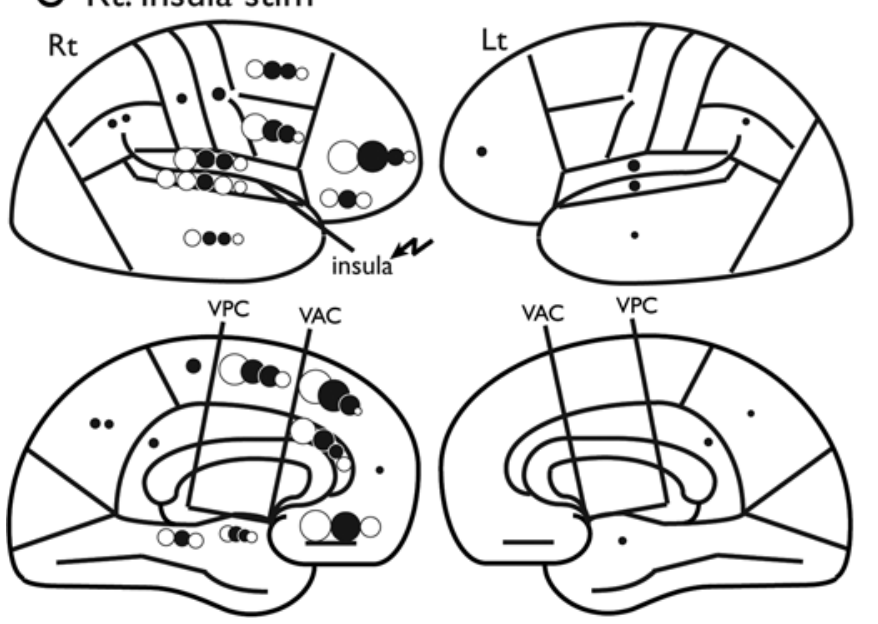

\section{B Lt. frontal operculum stim}
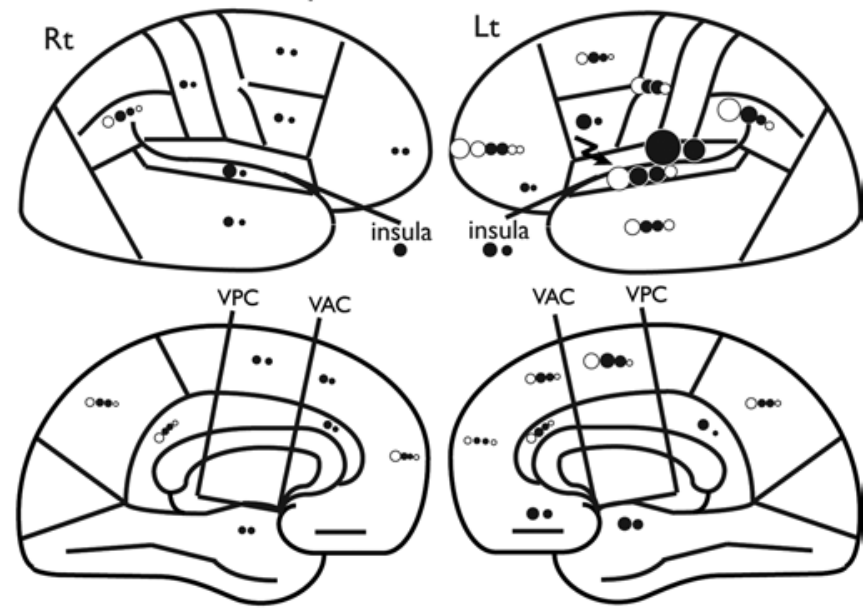

FIG. 4. Circle maps of RMS values of CCEPs in the frontal operculum stimulation $(A)$ right $(N=6)$ and $(B)$ left $(N=6)$, and anterior insula stimulation (C) right $(\mathrm{N}=5)$ and $(\mathrm{D})$ left $(\mathrm{N}=4)$. The diameter of the circle represents the RMS value of CCEP activities.

Blank parcellation (empty circles) indicate "not available" and black circles indicate the median value of RMS in each parcellation.

network around the insula and frontal operculum., ${ }^{7,25}$ The frontoinsular $\mathrm{U}$ fibers have been shown to connect various regions of the frontal operculum with the anterior insular cortex, ${ }^{7}$ which may reflect the connections between the frontal operculum and insula observed in our study. Several connections beneath the insula, including the uncinate fasciculus and extreme capsule, link temporal regions with the PFC. ${ }^{25}$ Therefore, frontotemporal CCEP responses may have been induced through these fibers in the insular stimulation. Previous CCEP study also revealed the anterior insular connectivity with the anterior frontotemporal brain region, which is almost consistent with our result. ${ }^{2}$ These networks may be involved in the anterior insular functions relevant for homeostasis or emotional processing. ${ }^{26}$ Posterior insular was not examined in the present study due to the limitations of electrode coverage. It is an interesting consideration for future analysis.

Our results showed that the vPM and SMA developed more in the network in the dominant hemisphere. This asymmetry in white-matter fibers remains controver- sial.5,17,27 However, several studies indicated the lateralization of the dominant hemispheric network associated with the vPM (e.g., the frontoparietal network ${ }^{5,7}$ ) and SMA (e.g., the frontal aslant tract ${ }^{7}$ ). These findings may lead to better hypotheses regarding higher language and motor functions in the dominant hemisphere and suggest the possibility that our method could detect the "functional" networks. Our sample size was small and the functional aspect was not evaluated in this study. Further analyses are necessary to confirm the interhemispheric asymmetry of connectivity, including large sample size and functional evaluation.

Several limitations must be acknowledged in this study. First, the neurophysiological mechanism of generating CCEP is still unclear and the possible pathway may include the monosynaptic and multisynaptic pathways. We cannot mention the precise pathway of each response in this study. Next, CCEPs may not enable full and comprehensive mapping of whole networks. The results of our study are limited by the restricted spatial coverage of the 
depth electrodes, the variable locations in selected areas, and the small number of these electrodes. SEEG is advantageous for sampling deep regions (e.g., insula, operculum, and medial aspect of hemisphere) and has relatively low complication rates; however, it has a limited ability in recording contiguous cortical regions. Conversely, strip electrodes are advantageous for exploring the superficial cortical areas. Consequently, these methods can be considered complementary and the combination of these techniques will probably provide better 3D cortical recordings and stimulation data. ${ }^{12}$

In addition, there are individual varieties of CCEP responses and locational variations of electrodes. CCEP responses and the location of electrodes varied among individuals, even though the electrodes were placed within the same anatomical areas, which may have affected the results obtained. Another possible issue is the effects of anticonvulsants on CCEP recordings. Patients were receiving anticonvulsants at the time of this study and the effects of these drugs on CCEP recordings remain unclear. Therefore, we cannot exclude the possibility that the effects of medication biased our CCEP results. The effects of epileptogenicity on the network also need to be considered. Furthermore, this study was performed in patients with intractable epilepsy. Thus, our results may have included an abnormal network that developed or was reorganized due to epileptogenicity.

Although CCEPs have several methodological limitations, they still provide significant information on human brain connectivity. A deeper understanding of functional connectivities in the brain will lead to the development of better treatments for patients with intractable focal epilepsies. Previously, we attempted to map the brain network and reported the connectivity pattern of limbic systems. ${ }^{13}$ A better understanding of the complicated network of the frontal and insular system will undoubtedly help the interpretation of semiology and seizure-spread patterns in patients with epilepsy arising within these areas.

\section{Conclusions}

We herein demonstrated functional (electrophysiological) connectivities in the human frontal and insular system, including its intrinsic reverberating circuits, both uniand bidirectional connections, and its extrinsic output to selected brain regions (Fig. 5). Each part had a different connectivity pattern, which may reflect each functional role. Further studies are needed to confirm these results and validate them in patients with epilepsy and other neuropsychiatric diseases.

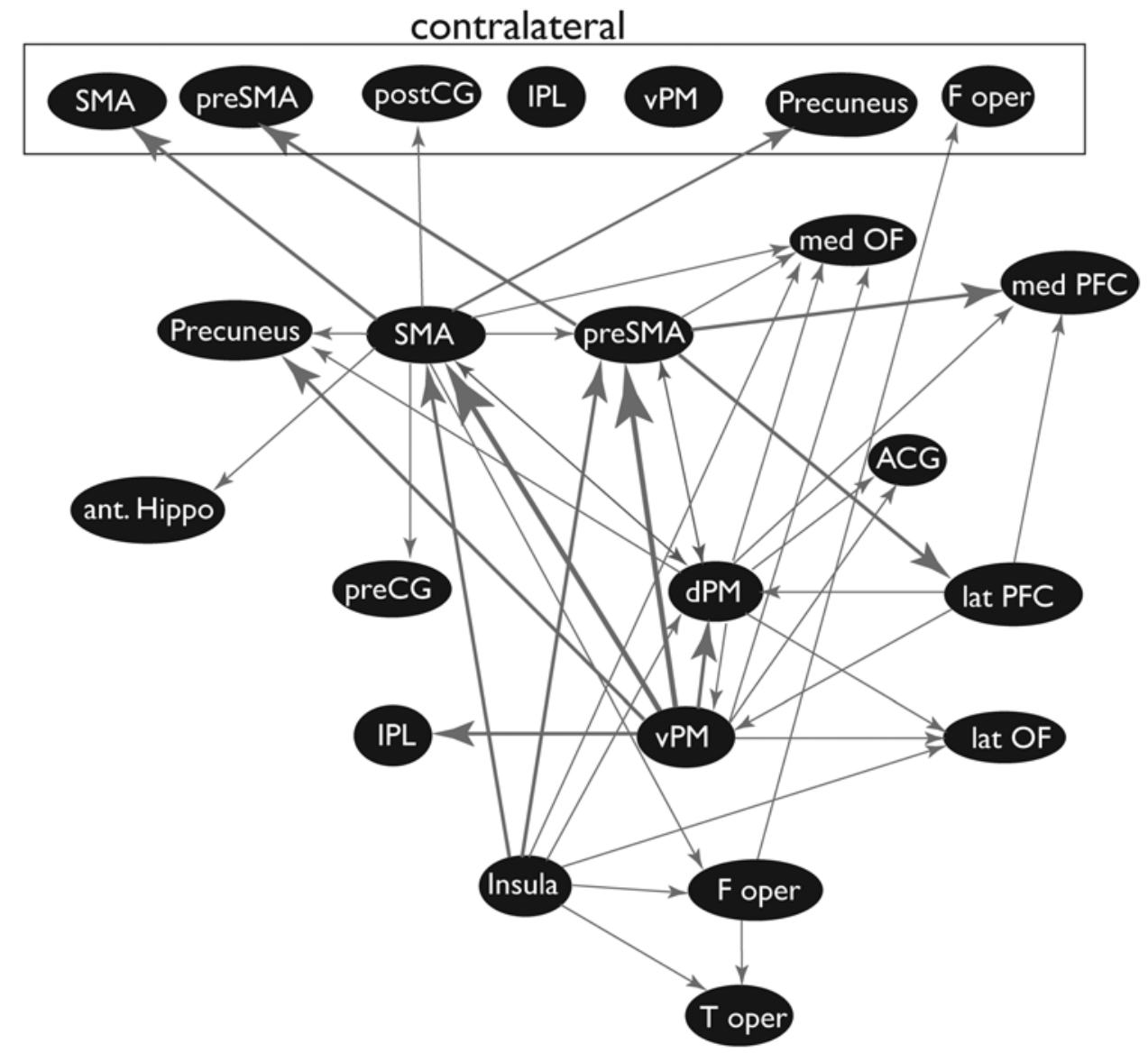

FIG. 5. Summary of areas in which stimulation elicited CCEP responses. ACG = anterior cingulate gyrus; $F=$ frontal; Hippo = hippocampus. 


\section{Acknowledgments}

We gratefully acknowledge Tim O'Connor, Karl Horning, and Zhe Piao for their technical assistance.

\section{References}

1. Alexopoulos AV, Tandon N: Basal frontal lobe epilepsy, in Lüders HO (ed): Textbook of Epilepsy Surgery. London: Informa Healthcare, 2008

2. Almashaikhi T, Rheims S, Jung J, Ostrowsky-Coste K, Montavont A, De Bellescize J, et al: Functional connectivity of insular efferences. Hum Brain Mapp 35:5279-5294, 2014

3. Bauer S, Hamer HM, Rosenow F: Premotor and central lobe epilepsy, in Lüders HO (ed): Textbook of Epilepsy Surgery. London: Informa Healthcare, 2008

4. Bleasel A, Dinner DS: Mesial frontal epilepsy, in Lüders HO (ed): Textbook of Epilepsy Surgery. London: Informa Healthcare, 2008

5. Büchel C, Raedler T, Sommer M, Sach M, Weiller C, Koch MA: White matter asymmetry in the human brain: a diffusion tensor MRI study. Cereb Cortex 14:945-951, 2004

6. Butti C, Hof PR: The insular cortex: a comparative perspective. Brain Struct Funct 214:477-493, 2010

7. Catani M, Dell'acqua F, Vergani F, Malik F, Hodge H, Roy P, et al: Short frontal lobe connections of the human brain. Cortex 48:273-291, 2012

8. Catani M, Thiebaut de Schotten M: A diffusion tensor imaging tractography atlas for virtual in vivo dissections. Cortex 44:1105-1132, 2008

9. Cheney PD: Role of cerebral cortex in voluntary movements. A review. Phys Ther 65:624-635, 1985

10. Dinner DS, Lüders HO: Cortical mapping by electrical stimulation of subdural electrodes: supplementary sensorimotor area in human, in Lüders HO (ed): Textbook of Epilepsy Surgery. London: Informa Healthcare, 2008

11. Enatsu R, Matsumoto R, Piao Z, O'Connor T, Horning K, Burgess RC, et al: Cortical negative motor network in comparison with sensorimotor network: a cortico-cortical evoked potential study. Cortex 49:2080-2096, 2013

12. Enatsu R, Bulacio J, Najm I, Wyllie E, So NK, Nair DR, et al: Combining stereo-electroencephalography and subdural electrodes in the diagnosis and treatment of medically intractable epilepsy. J Clin Neurosci 21:1441-1445, 2014

13. Enatsu R, Gonzalez-Martinez J, Bulacio J, Kubota Y, Mosher J, Burgess RC, et al: Connections of the limbic network: a corticocortical evoked potentials study. Cortex 62:20-33, 2015

14. Freund HJ: Functional organization of the human supplementary motor area and dorsolateral premotor cortex. Adv Neurol 70:263-269, 1996

15. Garzon E, Lüders HO: Cingulate epilepsy, in Lüders HO (ed): Textbook of Epilepsy Surgery. London: Informa Healthcare, 2008

16. Isnard J, Ryvlin P, Mauguière F: Insular epilepsy, in Lüders HO (ed): Textbook of Epilepsy Surgery. London: Informa Healthcare, 2008

17. Iwabuchi SJ, Häberling IS, Badzakova-Trajkov G, Patston LL, Waldie KE, Tippett LJ, et al: Regional differences in cerebral asymmetries of human cortical white matter. Neuropsychologia 49:3599-3604, 2011

18. Krawczyk DC: Contributions of the prefrontal cortex to the neural basis of human decision making. Neurosci Biobehav Rev 26:631-664, 2002

19. Kubota Y, Enatsu R, Gonzalez-Martinez J, Bulacio J, Mosher
J, Burgess RC, et al: In vivo human hippocampal cingulate connectivity: a corticocortical evoked potentials (CCEPs) study. Clin Neurophysiol 124:1547-1556, 2013

20. Kucukyuruk B, Richardson RM, Wen HT, Fernandez-Miranda JC, Rhoton AL Jr: Microsurgical anatomy of the temporal lobe and its implications on temporal lobe epilepsy surgery. Epilepsy Res Treat 2012:769825, 2012

21. Lacruz ME, García Seoane JJ, Valentin A, Selway R, Alarcón G: Frontal and temporal functional connections of the living human brain. Eur J Neurosci 26:1357-1370, 2007

22. Matsumoto R, Nair DR, LaPresto E, Najm I, Bingaman W, Lüders HO: Cortico-cortical evoked potentials, in Lüders HO (ed): Deep Brain Stimulation and Epilepsy. London: Martin Dunitz, 2004

23. Matsumoto R, Nair DR, LaPresto E, Bingaman W, Shibasaki $\mathrm{H}$, Lüders HO: Functional connectivity in human cortical motor system: a cortico-cortical evoked potential study. Brain 130:181-197, 2007

24. Nair DR, Burgess R, McIntyre CC, Lüders H: Chronic subdural electrodes in the management of epilepsy. Clin Neurophysiol 119:11-28, 2008

25. Schmahmann JD, Pandya DN, Wang R, Dai G, D'Arceuil HE, de Crespigny AJ, et al: Association fibre pathways of the brain: parallel observations from diffusion spectrum imaging and autoradiography. Brain 130:630-653, 2007

26. Stephani C, Fernandez-Baca Vaca G, Maciunas R, Koubeissi M, Lüders HO: Functional neuroanatomy of the insular lobe. Brain Struct Funct 216:137-149, 2011

27. Watkins KE, Paus T, Lerch JP, Zijdenbos A, Collins DL, Neelin P, et al: Structural asymmetries in the human brain: a voxel-based statistical analysis of 142 MRI scans. Cereb Cortex 11:868-877, 2001

28. Wilson CL, Isokawa M, Babb TL, Crandall PH: Functional connections in the human temporal lobe. I. Analysis of limbic system pathways using neuronal responses evoked by electrical stimulation. Exp Brain Res 82:279-292, 1990

\section{Disclosures}

Dr. Nair is a consultant for Brain Sentinel and NeuroPace. Dr. Najm is a consultant for UCB and Sunovion.

\section{Author Contributions}

Conception and design: Nair, Enatsu, Gonzalez-Martinez, Burgess, Najm. Acquisition of data: Nair, Enatsu, GonzalezMartinez, Najm. Analysis and interpretation of data: Nair, Enatsu, Bulacio, Mosher, Burgess, Najm. Drafting the article: Enatsu. Reviewed submitted version of manuscript: Nair, Enatsu, Gonzalez-Martinez, Bulacio, Burgess. Administrative/technical/material support: Gonzalez-Martinez, Bulacio, Mosher, Burgess, Najm. Study supervision: Nair, Gonzalez-Martinez, Burgess, Najm.

\section{Supplemental Information}

Previous Presentations

Portions of this work were presented in poster form at the 68th Annual Meeting of the American Epilepsy Society, Seattle, Washington, December 6, 2014.

\section{Correspondence}

Dileep R. Nair, Epilepsy Center, Neurological Institute, Cleveland Clinic, S51, 9500 Euclid Ave., Cleveland, OH 44195. email: naird@ccf.org. 